\title{
Recurrent Bilateral Breast Abscess in Immunocompetent Women: A Case Report and Literature Review
}

\author{
Hiyam AL Haddad ${ }^{1 *}$, Hend AL Shamsi ${ }^{2}$ and Najla AL Askar ${ }^{2}$ \\ ${ }^{1}$ Department of surgery, King Fahad Hospital of the University, Saudi Arabia \\ ${ }^{2}$ Imam Abdulrahman Bin Faisal University, Saudi Arabia
}

Received: February 15, 2018; Published: March 26, 2018

*Corresponding author: Hiyam Al Haddad, Consultant Breast surgeon, Department of surgery, King Fahd hospital of the University, Saudi Arabia, Email:dr_hiyam@yahoo.com

\section{Introduction}

Breast abscess is a localized pocket of infection containing pus tissue; that commonly affects women of reproductive years with age ranging from $18-50$ years. It is divided into a lactation and non-lactation infection. It can affect overlying skin as a primary event or secondary to a lesion such as sebaceous cyst [1]. Breast abscesses are typically caused by bacteria, most commonly Staphylococcus aureus. Other causes include mycobacterial and fungal infections [2]. There have been few reports of different infections caused by Salmonella spp. in recent years and a single case of bilateral breast abscess caused by E. coli and few by Proteus mirabilis, to our knowledge, there were no cases reported with recurrent breast abscesses caused by Kocuria kristinae. We present an unusual case of recurrent bilateral breast abscess caused by multiple organisms in an immunocompetent non-lactating patient.

\section{Case Report}

A 41-year-old Non Diabetic Saudi single female presented through the emergency department October 2015 complaining of progressive breast pain for 15 days' duration and a lump in the left breast. The patient denied history of fever, nipple or skin changes. On physical examination, she was cachectic and had left breast erythema, and tender mass. Admitted under the impression of mastitis with abscess. Further history taking was insignificant (negative history of fever, night sweats, smoking, alcohol or illicit drug use, trauma, nipple piercing, insect bite, nor swimming in public pools), apart from repeated visit to GIT clinic for weight loss and anorexia. She also denied history of ever being sexually active. In her family history, her aunt suffered from breast cancer. Laboratory investigations revealed elevated white blood cells (lymphocytes), total bilirubin, \& ESR.
She was managed with IV antibiotic \& incision drainage done. The culture isolated Pseudomonas aeruginosa (Table 1).

Table 1: From October 2015 till January 2018 patient admitted ten times with repeated bilateral breast abscess.

\begin{tabular}{|c|c|c|}
\hline October 2015 & LT breast & $\begin{array}{c}\text { E coli/Pseudomonas } \\
\text { aeruginosa }\end{array}$ \\
\hline November 2015 & RT + LT Breasts & E coli/Proteus mirabilis \\
\hline January 2016 & RT Breast & $\begin{array}{c}\text { E coli/Pseudomonas } \\
\text { aeruginosa }\end{array}$ \\
\hline September 2016 & LT Breast & E coli/ Proteus mirabilis \\
\hline September 2016 & RT Breast & $\begin{array}{c}\text { Ecoli/Kocuria ristinae/ } \\
\text { Salmonella }\end{array}$ \\
\hline July 2017 & RT + LT Breast & Mixed organisms \\
\hline July 2017 & LT + RT Breast & Proteus mirabilis \\
\hline August 2017 & LT Breast & Proteus mirabilis \\
\hline November 2017 & RT Breast & E coli/Proteus mirabilis \\
\hline January 2018 & RT Breast & $\begin{array}{c}\text { KlebsiellaPneumoniae/ } \\
\text { Citrobacter species }\end{array}$ \\
\hline
\end{tabular}

She noted flu like symptoms prior to each attack. She noticed that her attacks were mostly when her weight decreases since she's underweight with history of weight loss and decreased appetite over the last few years. Her height is $156.5 \mathrm{~cm}$ and her weight varies between $37-47 \mathrm{~kg}$ making her average $\mathrm{BMI}=17$ which is considered underweight (She currently weighs $38 \mathrm{~kg}$ ). She was investigated thoroughly for the decrease in appetite and weight loss and nothing found except positive $\mathrm{H}$ Pylori stool antigen each time. Hepatitis profile, HIV test, and TB tests were negative as well as cancer screening. IgG, IgM, IgE, are normal level. Anti endomysial antibodies and Antigliadin antibodies are negative. Breast biopsy was negative for cancer or chronic inflammatory condition. 


\section{Discussion}

The main concern of most of the women presenting with breast symptoms, including lumps, is likelihood of cancer, however, most breast masses are benign including fibrodenomas, fibrocystic condition, intraductal papilloma and breast abscesses [1]. WHO report on mastitis in 2000 clearly indicates that mastitis and abscesses are more common in lactating versus non-lactating women and NHS choices website article on breast abscesses, stated that "Breast abscesses are very uncommon in women who are not producing milk." Non-lactational breast abscesses can be divided into two broad groups, Peripheral and Areolar.

Breast abscess in non-lactating women had limited data in the literature [4]. the most significant risk factor for nonlactating breast abscess is diabetes mellitus (DM), smoking and immune suppression which was not identified as a risk factors in our case. Granulomatous mastitis another underlying diagnosis with high recurrence rate. Most common microorganism isolated from breast abscess is Staph [21-23]. Aureus other rare bacteria species can be found in immunocompromised patent. In our case, the identifiable organisms were Salmonella sp., Pseudomonas arignosa, E. coli, Proteus mirabilis, Klebsiella pneumoniae, Citrobacter species and Kocuria kristinae in different occasions.

Salmonella typhi bacteremia is occasionally associated with extra intestinal disease. It is capable of forming abscesses in various organs such as liver, subcutaneous tissue, muscles and skin. The pathogenesis of abscess formation is not well established [24]. The possible causes may be infective bile from carriers, hematogenous spread from distant site, and lym $\urcorner$ phatic spread from gastrointestinal tract. Bilateral breast ab-scesses due to Salmonella typhi are a rare presentation. However, the present case was not associated with a detectable bacteremia in the past [3]. It is well known that the most common cause of community-acquired urinary tract infection is E. coli.

However, it is a rare occurrence of a breast abscess caused by E. coli. Proteus mirabilis is most often associated with infections of the genitourinary tract. Breast abscess formation with Proteus mirabilis is uncommon. This infection is more common in diabetic patients who also have an increased risk of abscess recurrence [9]. The treatment of breast abscess during the nonlactating period is controversial in the literature, especially regarding such rare infections. For an early abscess presenting as an indurate mass, a course of antibiotics may suffice. For a mature abscess (e.g., fluctuant mass), surgical intervention along with antibiotics is indicated. The following table summarizes literature review of 24 cases of breast abscess of Non Staph [25]. Aureus rare microorganisms in Non lactating woman, however, almost all of them have identifiable risk factors which lack in our case (Table 2).

Table2: Literature review of 24 cases of breast abscess.

\begin{tabular}{|c|c|c|c|c|c|c|c|}
\hline S. No: & Name & Age & Gender & Organism & Side & $\begin{array}{l}\text { Co-morbidities/ } \\
\text { risk factors }\end{array}$ & $\begin{array}{l}\text { Important } \\
\text { Information }\end{array}$ \\
\hline 1 & $\begin{array}{l}\text { Bilateral Breast } \\
\text { Abscess Caused by } \\
\text { E. coli in a Non- } \\
\text { lactating Woman: A } \\
\text { Rare Case [6] }\end{array}$ & 36 & Female & E. coli & bilateral & $\begin{array}{c}\text { She had been } \\
\text { diagnosed with } \\
\text { urinary tract } \\
\text { infection about } 10 \\
\text { days ago }\end{array}$ & \\
\hline 2 & $\begin{array}{c}\text { A Rare Case of } \\
\text { Tubercular Breast } \\
\text { Abscess in a Young } \\
\text { Immunocompetent } \\
\text { ssNon- Lactating } \\
\text { Female [8] }\end{array}$ & 22 & Female & M. Tuberculosis & Right & $\begin{array}{l}\text { Patient is a resident } \\
\text { at an endemic area }\end{array}$ & $\begin{array}{l}\text { Patient had history of } \\
\text { intermittent fever, loss } \\
\text { of appetite and loss of } \\
\text { weight for 5-6 weeks } \\
\text { prior to presentation }\end{array}$ \\
\hline 3 & $\begin{array}{c}\text { A Rare Case of } \\
\text { Breast Abscess Due } \\
\text { To Salmonella Typhi } \\
\text { [3] }\end{array}$ & 60 & Female & $\begin{array}{l}\text { Salmonella typhi growth } \\
\text { sensitive to Amikacin/ } \\
\text { Ampicilinsulbactam/ Cefepime/ } \\
\text { Ceftazidime/Pipataz/Imipenam }\end{array}$ & Right & $\begin{array}{c}\text { Patient was } \\
\text { a diabetic on } \\
\text { irregular treatment }\end{array}$ & \\
\hline 4 & $\begin{array}{l}\text { Bilateral Breast } \\
\text { Abscesses due to } \\
\text { Salmonella enterica } \\
\text { Serotype Typhi [9] }\end{array}$ & 29 & Female & $\begin{array}{l}\text { Salmonella typhi sensitive to } \\
\text { chloramphenicol, cefotaxime, } \\
\text { ciprofloxacin, and co-trimoxazole } \\
\text { but resistant to ampicillin and } \\
\text { nalidixic acid }\end{array}$ & Bilateral & $\begin{array}{l}\text { It was associated } \\
\text { with a detectable } \\
\text { bacteremia in the } \\
\text { past }\end{array}$ & \\
\hline 5 & $\begin{array}{c}\text { Bilateral brucellar } \\
\text { breast abscess in a } \\
\text { 48-year-old woman } \\
\text { [10] }\end{array}$ & 48 & Female & Brucella species & Bilateral & $\begin{array}{c}\text { Patient was } \\
\text { involved in } \\
\text { stockbreeding on } \\
\text { a farm }\end{array}$ & $\begin{array}{l}\text { Patient had history of } \\
\text { night sweats for the } \\
\text { previous } 2 \text { years }\end{array}$ \\
\hline
\end{tabular}




\begin{tabular}{|c|c|c|c|c|c|c|c|}
\hline 6 & $\begin{array}{l}\text { Breast abscess due } \\
\text { to Actinomyces } \\
\text { europium [11] }\end{array}$ & 67 & Female & $\begin{array}{l}\text { Actinomyces (A. europaeus) and a } \\
\text { mixed anaerobic flora composed } \\
\text { of Gram-negative rods and Gram- } \\
\text { positive cocci that were not } \\
\text { further characterized }\end{array}$ & Left & None identified & $\begin{array}{l}\text { Patient had undergone } \\
\text { uncomplicated open } \\
\text { heart surgery to receive } \\
\text { a prosthetic aortic } \\
\text { valve for severe aortic } \\
\text { stenosis } 2 \text { months prior }\end{array}$ \\
\hline 7 & $\begin{array}{l}\text { Breast abscess due } \\
\text { to Actinomyces } \\
\text { europaeus [12] }\end{array}$ & 43 & Female & $\begin{array}{l}\text { Salmonella enterica } \\
\text { serotype Typhimurium. The } \\
\text { microorganism was susceptible } \\
\text { to ampicillin, ciprofloxacin, } \\
\text { trimethoprimsulfamethoxazole, } \\
\text { erythromycin, chloramphenicol, } \\
\text { ceftazidime, and ceftriaxone. }\end{array}$ & Right & $\begin{array}{l}\text { The patient had a } \\
\text { history of } 5 \text { years } \\
\text { of RA and was on } \\
\text { prednisone therapy. }\end{array}$ & $\begin{array}{l}\text { The patient had history } \\
\text { of diarrhoea for } \\
\text { approximately } 3 \text { days } \\
\text { and did not receive any } \\
\text { antibiotic treatment } \\
2 \text { months prior to } \\
\text { presentation }\end{array}$ \\
\hline 8 & $\begin{array}{c}\text { Breast Abscess } \\
\text { in a Man Due to } \\
\text { Salmonella enterica } \\
\text { Serotype Enteritidis } \\
\text { [13] }\end{array}$ & 70 & Male & $\begin{array}{l}\text { Salmonella enterica serotype } \\
\text { Enteritidis sensitive to } \\
\text { amoxicillin-clavulanic acid, } \\
\text { ceftriaxone, trimethoprim- } \\
\text { sulfamethoxazole, and } \\
\text { ciprofloxacin }\end{array}$ & Left & $\begin{array}{l}\text { Patient is non- } \\
\text { insulin-dependent } \\
\text { diabetic }\end{array}$ & $\begin{array}{l}\text { The patient had } \\
\text { history of an episode } \\
\text { of severe acute } \\
\text { gastroenterocolitis } 10 \\
\text { months earlier with } \\
\text { signs of acute renal } \\
\text { failure }\end{array}$ \\
\hline 9 & $\begin{array}{l}\text { Brucellar breast } \\
\text { abscess [14] }\end{array}$ & 63 & Female & Brucella & Left & $\begin{array}{l}\text { Patient had } \\
\text { a history of } \\
\text { unpasteurized } \\
\text { cheese } \\
\text { consumption }\end{array}$ & \\
\hline 10 & $\begin{array}{l}\text { Fusarium solani } \\
\text { Breast Abscess [15] }\end{array}$ & 55 & Female & Fusarium solani & Right & $\begin{array}{l}\text { Patient is a diabetic } \\
\text { on irregular therapy } \\
\text { for } 6 \text { years }\end{array}$ & $\begin{array}{l}\text { Patient worked in } \\
\text { paddy field and carried } \\
\text { hay bundles between } \\
\text { her right arm and } \\
\text { her breast. Fusarium } \\
\text { solani spores from the } \\
\text { hay probably gained } \\
\text { entry through minor } \\
\text { abrasions sustained } \\
\text { while working in the } \\
\text { field }\end{array}$ \\
\hline 11 & $\begin{array}{l}\text { Cordylobia rodhaini } \\
\text { infestation of the } \\
\text { breast: Report of a } \\
\text { case mimicking a } \\
\text { breast abscess [16] }\end{array}$ & 17 & Female & Cordylobia rodhaini Parasite. & Left & $\begin{array}{l}\text { The patient } \\
\text { returned from a } \\
\text { voyage in Ghana } \\
\text { fourteen days } \\
\text { prior to the first } \\
\text { presentation }\end{array}$ & \\
\hline 12 & $\begin{array}{l}\text { First report of } \\
\text { Actinomyces } \\
\text { europaeus } \\
\text { bacteraemia result } \\
\text { from a breast } \\
\text { abscess in a 53-year- } \\
\text { old man [17] }\end{array}$ & 53 & Male & $\begin{array}{c}\text { Actinomyces europaeus and } \\
\text { Porphyromonas }\end{array}$ & Right & $\begin{array}{c}\text { The patient was } \\
\text { diabetic and } \\
\text { smoked } 20-30 \\
\text { cigarettes a day. } \\
\text { She had recurrent } \\
\text { cutaneous } \\
\text { abscesses, mainly in } \\
\text { the axillae and the } \\
\text { groin }\end{array}$ & $\begin{array}{c}\text { Other identified } \\
\text { comorbidities } \\
\text { were obesity, } \\
\text { chronic obstructive } \\
\text { pulmonary disease and } \\
\text { schizophrenia }\end{array}$ \\
\hline 13 & $\begin{array}{c}\text { Identification of } \\
\text { Propionibacterium } \\
\text { avidum from a } \\
\text { breast abscess: } \\
\text { an overlooked } \\
\text { etiology of clinically } \\
\text { significant infections } \\
{[18]}\end{array}$ & 37 & Female & $\begin{array}{l}\text { Propionibacterium avidum } \\
\text { sensitive to amoxicillin, } \\
\text { amoxicillin-clavulanic acid, } \\
\text { ceftriaxone and levofloxacin and } \\
\text { resistant to clindamycin. }\end{array}$ & Right & $\begin{array}{l}\text { The patient } \\
\text { had undergone } \\
\text { bilateral reduction } \\
\text { mammoplasty } \\
\text { for symptomatic } \\
\text { hypertrophy }\end{array}$ & \\
\hline 14 & $\begin{array}{l}\text { Male Breast Abscess } \\
\text { Secondary to } \\
\text { Actinomycosis: A } \\
\text { Case Report [19] }\end{array}$ & 24 & male & Actinomycosis neuii & Left & $\begin{array}{l}\text { Medical history } \\
\text { of Human } \\
\text { Immunodeficiency } \\
\text { Virus (HIV) and } \\
\text { syphilis }\end{array}$ & $\begin{array}{l}\text { The patient underwent } \\
\text { bilateral reduction } \\
\text { mammoplasty for } \\
\text { gynecomastia eight } \\
\text { years previously. }\end{array}$ \\
\hline
\end{tabular}




\section{Open Access Journal of Surgery}

\begin{tabular}{|c|c|c|c|c|c|c|c|}
\hline 15 & $\begin{array}{l}\text { Melioidosis breast } \\
\text { abscesses [20] }\end{array}$ & 46 & Female & Burkholderia pseudomallei & Right & $\begin{array}{l}\text { Travelling to Aruba } \\
\text { and direct contact } \\
\text { with an iguana and } \\
\text { multiple mosquito } \\
\text { bites }\end{array}$ & $\begin{array}{l}\text { Past medical history of } \\
\text { recurrent breast cysts, } \\
\text { which resolve on their } \\
\text { own }\end{array}$ \\
\hline 16 & $\begin{array}{l}\text { Mycobacterium } \\
\text { Fortuitum and } \\
\text { Anaerobic Breast } \\
\text { Abscess Following } \\
\text { Nipple Piercing: } \\
\text { Case Presentation } \\
\text { and Review of the } \\
\text { Literature [21] }\end{array}$ & 17 & Female & $\begin{array}{l}\text { Mycobacterium Fortuitum } \\
\text {-Prevotalla melanogenica }\end{array}$ & Right & Nipple piercing & \\
\hline 17 & $\begin{array}{c}\text { Mycobacterium } \\
\text { fortuitum breast } \\
\text { abscess after nipple } \\
\text { piercing [22] }\end{array}$ & 21 & Female & $\begin{array}{l}\text { Mycobacterium fortuitum } \\
\text {-nocardia species }\end{array}$ & Left & $\begin{array}{l}\text { Nipple piercing and } \\
\text { swimming in algae } \\
\text { water }\end{array}$ & \\
\hline 18 & $\begin{array}{l}\text { Periprosthetic } \\
\text { Breast Abscess } \\
\text { Caused by } \\
\text { Streptococcus } \\
\text { pyogenes After } \\
\text { Scarlet Fever [23] }\end{array}$ & 32 & Female & Streptococcus pyogenes (GAS) & Right & $\begin{array}{l}\text { History of breast } \\
\text { reconstruction with } \\
\text { implant and scarlet } \\
\text { fever }\end{array}$ & $\begin{array}{l}\text { History of mammary } \\
\text { Paget disease with } \\
\text { high-grade ductal } \\
\text { carcinoma in situ } \\
\text { (DCIS G3), treated } \\
\text { with right mastectomy } \\
\text { and axillary node } \\
\text { dissection, underwent } \\
\text { an immediate breast } \\
\text { reconstruction by } \\
\text { insertion of 350-mL } \\
\text { saline textured McGhee } \\
\text { style } 133 \text { LV implant. } \\
\text {-2 months later, she } \\
\text { developed fever and } \\
\text { sore throat, } 2 \text { days } \\
\text { later, she developed a } \\
\text { rash on the neck and } \\
\text { chest. After laboratory } \\
\text { investigations, she } \\
\text { was diagnosed with } \\
\text { scarlet fever and treated } \\
\text { accordingly }\end{array}$ \\
\hline 19 & $\begin{array}{l}\text { Proteus mirabilis } \\
\text { Breast Abscess A } \\
\text { Case Report and } \\
\text { Review of the } \\
\text { Literature [24] }\end{array}$ & 43 & Female & Proteus mirabilis & Right & $\begin{array}{c}\text { History of } \\
\text { chemotherapy, } \\
\text { hypertension, } \\
\text { smoking 7-pack- } \\
\text { year; she drank } \\
\text { alcohol } 2 \text { days a } \\
\text { week. - She felt her } \\
\text { boyfriend's poor } \\
\text { dentition had a role } \\
\text { in the development } \\
\text { of this infection }\end{array}$ & $\begin{array}{c}\text { History of } \\
\text { adenocarcinoma of } \\
\text { the colon status post } \\
\text { hemicolectomy and } 6 \\
\text { cycles of chemotherapy }\end{array}$ \\
\hline 20 & $\begin{array}{c}\text { Recurrent } \\
\text { Corynebacterium } \\
\text { kroppenstedtii } \\
\text { Breast Abscess in a } \\
\text { Young Asian Female } \\
\text { [25] }\end{array}$ & 36 & Female & Corynebacterium kroppenstedtii & Right & $\begin{array}{c}\text { History of mild } \\
\text { trauma (collision } \\
\text { with her 2-year-old } \\
\text { child) }\end{array}$ & $\begin{array}{c}\text { History of } \\
\text { hemithyroidectomy for } \\
\text { benign thyroid nodules.- } \\
\text { She had recurrent } \\
\text { breast abscess for } 3 \\
\text { times }\end{array}$ \\
\hline 21 & $\begin{array}{l}\text { Salmonella enterica } \\
\text { serovar Typhi in } \\
\text { breast abscess: A } \\
\text { case report [26] }\end{array}$ & 60 & Female & Salmonella typhi & $\begin{array}{c}\text { Not } \\
\text { noted }\end{array}$ & Diabetes & $\begin{array}{l}\text { There was no past } \\
\text { history of chest pain or } \\
\text { abdominal pain }\end{array}$ \\
\hline
\end{tabular}




\begin{tabular}{|c|c|c|c|c|c|c|c|}
\hline 22 & $\begin{array}{c}\text { Salmonella typhi } \\
\text { Breast Abscess: } \\
\text { An Uncommon } \\
\text { Manifestation of an } \\
\text { Uncommon Disease } \\
\text { in the United States } \\
\text { [27] }\end{array}$ & 28 & Female & Salmonella typhi & Right & $\begin{array}{l}\text { Eating undercooked } \\
\text { chicken several } \\
\text { weeks' prior } \\
\text { presentation at } \\
\text { a take-out place, } \\
\text { But denied any } \\
\text { gastrointestinal } \\
\text { symptoms }\end{array}$ & $\begin{array}{l}\text { She denied recent } \\
\text { trauma but had a } \\
\text { penetrating stick injury } \\
\text { to the right breast as a } \\
\text { child. }\end{array}$ \\
\hline 23 & $\begin{array}{c}\text { Salmonella typhi } \\
\text { Breast Abscess: } \\
\text { An Uncommon } \\
\text { Manifestation of an } \\
\text { Uncommon Disease } \\
\text { in the United States } \\
\text { [7] }\end{array}$ & 52 & Female & $\begin{array}{c}\text { Corynebacterium xerosis. } \\
\text {-Staphylococcus aureus. -Proteus } \\
\text { mirabillis. - Streptococcus } \\
\text { viridans }\end{array}$ & $\begin{array}{l}\text { Not } \\
\text { noted }\end{array}$ & $\begin{array}{l}\text { Wegener's } \\
\text { granulomatosis }\end{array}$ & $\begin{array}{c}5 \text { recurrent } \\
\text { episode of breast } \\
\text { abscess.- Idiopatic } \\
\text { granulomatous } \\
\text { mastitis was the } \\
\text { suspicious diagnosis } \\
\text { and a mastectomy } \\
\text { was performed.- Two } \\
\text { weeks later, the patient } \\
\text { showed a widespread } \\
\text { erythematous and } \\
\text { petechial vasculitic } \\
\text { rash with areas of } \\
\text { confluence together } \\
\text { with well-demarcated } \\
\text { areas of ulceration } \\
\text { affecting her legs. She } \\
\text { referred throat pain } \\
\text { and ear ache. Further } \\
\text { investigations findings } \\
\text { were Compatible } \\
\text { with Wegener's } \\
\text { granulomatosis }\end{array}$ \\
\hline 24 & $\begin{array}{c}\text { Breast abscess with } \\
\text { lethal septicemia } \\
\text { due to Pseudomonas } \\
\text { aeruginosa in a } \\
\text { patient with AIDS } \\
\text { [28] }\end{array}$ & 21 & Female & Pseudomonas aeruginosa & $\begin{array}{l}\text { Not } \\
\text { noted }\end{array}$ & HIV & $\begin{array}{l}\text { HIV infection had been } \\
\text { confirmed } 6 \text { years } \\
\text { earlier }\end{array}$ \\
\hline
\end{tabular}

\section{Conclusion}

Any case of a recurrent breast abscess in a non-lactating female must be heavily evaluated, in terms of clinical, laboratory and imaging assessment for any possible under lying risk factors can be identified. Keeping in mind the absence of such risk factors does not rule out rare or recurrent breast infections as it is the case in our patient whom after thorough investigation we could not find any identifiable risk factor.

The combination of medical and surgical management helps in such a case when supported by a microbiological culture and sensitivity report.

\section{References}

1. Dixon JM (1994) ABC of breast diseases. Breast infection BMJ 309 (6959): 946-949.

2. Nancy F, Crum Cianflone, Fadi A, Haddad, George Sakoulas (2013) A Breast Abscess, an Unusual Manifestation of Coccidioidomycosis? Infect Dis Clin Pract 21(4): 263-264.

3. Murugesan N, Alagar Samy R, Viswanathan MS, Anbazhagan A (2016) A rare case of breast abscess due to salmonella typhi. Int J Cur Res Rev 8(5): 10-12.

4. Rizzo M, Gabram S, Staley C, Peng L, Frisch A, et al. (2010) Management of breast abscesses in nonlactating women. Am Surg 76(3): 292-295.
5. Şimşek, G, Gündeș E, Tekin Ş, Tavlı Ş (2014) Bilateral Breast Abscess Caused by E. coli in a Non-lactating Woman: A Rare Case. J Breast Health 10(3): 174-176.

6. Malhotra S, Sharma S, Bhatia NJK, Sharma S, Hans C (2015) A Rare Case of Tubercular Breast Abscess in a Young Immunocompetent Non-Lactating Female. Clin Microbiol 4: 190.

7. Singh G, Dasgupta M, Gautam V, Behera A, Ray P (2011) Bilateral breast abscesses due to Salmonella enterica serotype typhi. J Global Infect Dis 3(4): 402-404.

8. Ibis C, Albayrak D, Yagci M (2009) Bilateral brucellar breast abscess in a 48-year-old woman. Annals of Saudi Medicine, 29(2): 158.

9. Silva WA, Pinheiro AM, Jahns B, Bo"gli-Stuber K, Droz S, et al. (2011) Breast abscess due to Actinomyces europaeus. Infection 39(3): 255258.

10. Irmak Baran, Neriman Aksu, Altan Aksoy (2016) Breast abscess due to Salmonella typhimurium in a patient with rheumatoid arthritis: a case report. BMC Infect Dis 16: 348.

11. Brnčić N, Gorup L, Strčić M, Abram M, Mustač E (2011) Breast Abscess in a Man Due to Salmonella enterica Serotype Enteritidis. J Clin Microbiol 50(1): 192-193.

12. Erdem G, Karakas H, Yetkin F, Alkan A, Firat A, et al. (2006) Brucellar breast abscess. Breast 15(4): 554-557.

13. Anandi V, Vishwanathan P, Sasikala S, Rangarajan M, Subramaniyan C, et al. (2005) Fusarium solani breast abscess. Indian J Med Microbiol 23(3): 198. 
14. Grassi V, Butterworth JW, Latiffi L (2016) Cordylobia rodhaini infestation of the breast: Report of a case mimicking a breast abscess. International Journal of Surgery Case Reports 27: 122-124.

15. Nielsen H (2015) First report of Actinomyces europaeus bacteraemia result from a breast abscess in a 53-year-old man. New Microbes New Infect 7: 21-22.

16. Kritikos A, Pagin M, Borens O, Voide C, Orasch C (2015) Identification of Propionibacterium avidum from a breast abscess: an overlooked etiology of clinically significant infections. New Microbes New Infect 4: 9-10.

17. Mahendiran SA, Leibman AJ, Kommehl AS (2016) Male Breast Abscess Secondary to Actinomycosis: A Case Report. J Clin Diagn Res 10(4): TD05-TD07.

18. Mickail N, Klein NC, Cunha BA, Schoch PA (2012) Melioidosis breast abscesses. Journal of Infection 64(4): 434-435.

19. Bengualid V, Singh V, Singh H, Berger J (2008) Mycobacterium fortuitum and Anaerobic Breast Abscess Following Nipple Piercing: Case Presentation and Review of the Literature. Journal of Adolescent Health 42(5): 530-532.

20. Abbass K, Adnan MK, Markert RJ, Emig M, Khan NA (2014) Mycobacterium fortuitum breast abscess after nipple piercing. Can Fam Physician 60(1): 51-52.

21. Persichetti P, Langella M, Marangi GF, Vulcano E, Gherardi G, et al (2008) Periprosthetic Breast Abscess Caused by Streptococcus pyogenes After Scarlet Fever. Ann Plast Surg 60(1): 21-23.

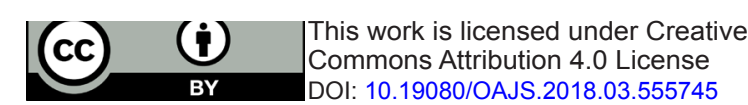

22. Labalo S, Saravolatz LD (2015) Proteus mirabilis Breast Abscess. Infectious Diseases in Clinical Practice 23(3): 126-127.

23. Goh Z, Tan AL, Madhukhumar P, Yong WS (2015) Recurrent Coryne bacterium kroppenstedtii Breast Abscess in a Young Asian Female. The Breast Journal 21(4): 431-432.

24. Kumar ES, Selvam RE, Ramesh SS (2012) Salmonella enterica serovar Typhi in breast abscess: A case report. Indian J Pathol Microbiol 55(4): 608-609.

25. Vikram Vattipally V, Thatigotla B, Nagpal K, Saraiya R, Henry M, et al. (2011) Salmonella typhi Breast Abscess: An Uncommon Manifestation of an Uncommon Disease in the United States. Am Surg 77: E133-135.

26. Comas $\mathrm{AG}$, Diana $\mathrm{CA}$, Crespo $\mathrm{CC}$, Cebollada MM, Liñán MA, et al. (2010) Wegener's granulomatosis presented as recurrent breast abscess. The Breast Journal 16(1): 82-84.

27. Rizzo M, Peng L, Frisch A, Jurado M, Umpierrez G (2009) Breast abscesses in nonlactating women with diabetes: clinical features and outcome. Am J Med Sci 338(2): 123-126.

28. Roca B, Vilar C, Pérez EV, Sáez Royuela A, Simón E (1996) Breast abscess with lethal septicemia due to Pseudomonas aeruginosa in a patient with AIDS. Presse Med 25(17): 803-804.

\section{Your next submission with Juniper Publishers will reach you the below assets}

- Quality Editorial service

- Swift Peer Review

- Reprints availability

- E-prints Service

- Manuscript Podcast for convenient understanding

- Global attainment for your research

- Manuscript accessibility in different formats ( Pdf, E-pub, Full Text, Audio)

- Unceasing customer service

Track the below URL for one-step submission https://juniperpublishers.com/online-submission.php 